\title{
ANÁLISIS DE LAS PRESTACIONES DE TERAPIA OCUPACIONAL INCLUIDAS EN EL RÉGIMEN DE GARANTÍAS EXPLÍCITAS EN SALUD (GES)
}

\author{
ANALYSIS OF OCCUPATIONAL THERAPY SERVICES INCLUDED IN THE REGIME OF \\ EXPLICIT HEALTH GUARANTEES (GES)
}

\section{Jean Gajardo'; Cristóbal Catalán²; Raquel Rioseco3; Isidora Vildósola ${ }^{4}$}

\begin{abstract}
RESUMEN
Introducción: La ley 19.966 establece un régimen de Garantías Explícitas en Salud que define el acceso, calidad, oportunidad y protección financiera de las prestaciones para el tratamiento de 80 patologías en Chile. Debido al alcance y la relevancia de esta modalidad institucional de atención en salud en un nivel nacional, es pertinente indagar en la incorporación de terapia ocupacional. Objetivo: Identificar las prestaciones de terapia ocupacional incluidas en el régimen de Garantías Explícitas en Salud al año 2017 y caracterizarlas según su frecuencia en los problemas de salud y según nivel de evidencia y grado de recomendación. Método: Revisión documental del Listado de Prestaciones Específicas versión 2016, y de las 80 Guías Clínicas Ges. Resultados: Se identificó una prestación de terapia ocupacional denominada "Atención Integral por Terapeuta Ocupacional". Dicha prestación se incluye en 12 problemas de salud según el Listado de Prestaciones Específicas. Se encontraron 10 Guías Clínicas que incorporan terapia ocupacional, con variados de grados de recomendación, mayormente Cy D. En relación con la cobertura de terapia ocupacional existe discordancia entre lo referido en Listado de Prestaciones Especificas y Guías Clínicas. Conclusiones: La terapia ocupacional tiene un limitado respaldo en evidencia en el sistema de Garantías Explícitas en Salud. Estos hallazgos refuerzan la necesidad de un mayor análisis de la evidencia de terapia ocupacional en los problemas de salud cubiertos por esta modalidad para fortalecer el diálogo con los tomadores de decisiones, y si es pertinente, continuar ampliando su cobertura garantizada a otros problemas de salud.
\end{abstract}

\section{PALAB RAS CLAVE}

Terapia ocupacional, políticas públicas, práctica clínica basada en la evidencia.

1 Terapeuta Ocupacional, Universidad de Chile, Máster en Gerontología Social, Universidad de León Doctor (c) en Salud Pública, Universidad de Chile. Departamento de Terapia Ocupacional y Ciencia de la Ocupación, Universidad de Chile. Researcher ID: I-7631-2013. Orcid ID: orcid.org/0000-0002-5699-0862. Independencia 1027, Independencia 8380453, Santiago jean.gajardo@uchile.cl.56229786342

2 Terapeuta Ocupacional, Universidad de Chile. Licenciado en Ciencias de la Ocupación Humana, Universidad de Chile. Servicio de Medicina Física y Rehabilitación, Hospital Clínico Universidad de Chile, Avenida Santos Dumont 999, Independencia 8380453, Santiago ccatalanaguila@gmail.com. Tel.: 56229788040.

3 Estudiante, Ayudante alumno de investigación. Escuela de Terapia Ocupacional, Universidad de Chile. Independencia 1027, Independencia 8380453, Santiago. r.elisabet.rioseco@hotmail.com. Tel.: 56229786342.

4 Terapeuta Ocupacional, Universidad de Chile. Licenciada en Ciencias de la Ocupación Humana, Universidad de Chile.Programa Más Adultos Mayores Autovalentes, comuna de Lampa. Servicio de Salud Metropolitano Norte. Ismael Carmona s/n, Lampa. Tel.: isivildosola@ gmail.com. Tel.: 56229786342 


\begin{abstract}
Introduction: Chilean Law 19.966 states a regime of Explicit Health Guarantees for access, quality, opportunity and economic protection of treatment coverage in 80 health problems. This law extents in a national level and is a milestone in the health care reform in Chile. However, there is no specific information on how occupational therapy is incorporated in this institutional modality of health coverage. Aim: To identify occupational therapy services included in the regime of Explicit Health Guarantees and describe services by frequency in health problems, and their levels of evidence and grades of recommendation. Methods: Documentary review of Specific Health Benefits 2016 List, and Clinical Practice Guides for 80 health problems covered by the Explicit Health Guarantees regime. Results: One service was identified, named "Integral Care by Occupational Therapy". This health service is included in 12 health problems referred in Specific Health Benefits 2016 List. Occupational therapy is included 10 Clinical Practice Guides, with differing grades of recommendation, mostly C and D. In relation to the incorporation of occupational therapy services, there is a mismatch between what is declared in the Specific Health Benefits 2016 List and the Clinical Guidelines. Conclusions: Occupational therapy has limited supporting evidence and grades of recommendation in the regime of Explicit Health Guarantees. These findings reinforce the need of a deeper analysis of the supporting evidence of occupational therapy for health problems covered by this policy, in order to strengthen the dialogue with policy makers and enable its coverage expansion to other health problems when corresponding.
\end{abstract}

\title{
KEYWORDS
}

Occupational therapy, public policy, health policy, evidence-based practice

Recibido: 20/09/2017

Aceptado: 9/11/2017 


\section{INTRODUCCIÓN}

En el año 2006, en el contexto de la reforma del Sistema de Salud Chile, se crea un sistema de garantías explícitas en salud (GES; Resolución N ${ }^{\circ}$ 60, 2007). Dicho sistema se enmarca en el Plan de Acceso Universal de Garantías Explícitas (o Plan AUGE), consistente, según lo establecido por la Ley $N^{\circ} 19.966$ (que lo denomina Régimen General de Garantías en Salud) en una reglamentación que garantiza el acceso a las acciones de promoción, protección y recuperación de la salud, en la cual se incorporan nuevos problemas de salud cada tres años.

El plan GES tiene por objetivo garantizar la cobertura de un número de problemas de salud por parte del Fondo Nacional de Salud (Fonasa) y las Instituciones de Salud Previsional (Isapres) asociando la distribución de recursos en salud para los problemas de salud de mayor morbilidad y mortalidad en el país, estableciendo su prioridad en la atención reflejada en cuatro garantías centrales: acceso, calidad, oportunidad y protección financiera (Bastías, Pantoja, Leisewitz \& Zárate, 2008). Actualmente, el plan GES considera la cobertura de 80 problemas de salud y forma parte de la modalidad de atención institucional del Sistema de Salud chileno.

El régimen GES ha permitido un relevante avance en la protección de derechos de salud, no obstante, durante su implementación han emergido críticas ligadas a las diferencias evaluadas entre las garantías según prestador público o privado (Henríquez, 2017; Pardo y Schott, 2014). También, se ha referido que existe un elevado desconocimiento por parte de los usuarios de Fonasa e Isapres sobre el funcionamiento de este sistema, y de las prestaciones específicas garantizadas por Ley (Henríquez, 2017), lo que se traduce en brechas de acceso y utilización de recursos.

El sistema GES se apoya de dos principales formas de documentación: un Listado de Prestaciones Específicas, y Guías de Clínicas para cada uno de los problemas de salud incluidos. El Listado de Prestaciones Específicas corresponde a la compilación de las acciones o prestaciones garantizadas por Ley para cada problema de salud, enfatizando en su dimensión administrativa. Su versión más reciente corresponde al año 2016 (Ministerio de Salud, 2016). Por su parte, las guías clínicas (GC), o Guías de Práctica Clínica (GPC), son enunciados elaborados con el fin de asistir las decisiones de los profesionales del área salud y a sus pacientes, acerca del cuidado apropiado en circunstancias específicas, que buscan optimizar las atenciones, basándose en la revisión sistemática de la evidencia y una evaluación de los beneficios y daños de opciones de manejo alternativas (Pantoja y Soto, 2014). El Listado de Prestaciones Específicas incorporadas para los problemas de salud GES debiese acompañarse de recomendaciones técnicas específicas para el diagnóstico y tratamiento en cada uno de los problemas de salud, a través de guías clínicas. Actualmente los 80 problemas de salud cuentan con GC, no obstante, su actualización es variable. Existen diferentes metodologías para el desarrollo de estas guías, con un consenso general sobre la necesidad de incluir, entre otros elementos, representantes de las diferentes disciplinas, quienes evalúan la pertinencia de la incorporación de prestaciones a partir de sus niveles de evidencia científica, grados de recomendación, y la propia experiencia clínica (Ministerio de Salud, 2014).

Con el propósito de promover buenas prácticas en salud que se sustenten en evidencia científica, se han desarrollado diferentes modelos de jerarquización de la investigación que informe dicha evidencia (Manterola, Asenjo-Lobos, \& Otzen, 2014). Estos modelos representan consensos generados para posibilitar análisis comparativos y facilitar la evaluación de la calidad de las investigaciones, para finalmente, orientar la toma de decisiones en salud. Por ejemplo, Sackett (1986) jerarquiza la evidencia en niveles que van de 1 a 5 , siendo el nivel 1 la "mejor evidencia" y el nivel 5 la "peor, la más mala o la menos buena", y establece una relación entre niveles de evidencia y sus consecuentes grados de recomendación en los diferentes ámbitos de la acción sanitaria, en los procesos de salud-enfermedad: terapia, prevención, etiología y daño; pronóstico; diagnóstico; y estudios económicos (FIGURA 1). 
FIGURA 1 JERARQUIZACIÓN DE GRADOS DE RECOMENDACIÓN Y NIVELES DE EVIDENCIA PARA INTERVENCIONES TERAPÉUTICAS Y PREVENTIVAS SEGÚN SACKETT

\begin{tabular}{|c|c|c|}
\hline GR & NE & \\
\hline \multirow[t]{2}{*}{ A } & $1 \mathrm{a}$ & $\begin{array}{l}\text { Revisión Sistemática de Ensayos } \\
\text { Controlados con Asignación Aleatoria. }\end{array}$ \\
\hline & $1 b$ & $\begin{array}{l}\text { Ensayo Controlado con Asignación } \\
\text { Aleatoria e intervalo de confianza } \\
\text { estrecho. }\end{array}$ \\
\hline \multirow[t]{4}{*}{ B } & $2 \mathrm{a}$ & $\begin{array}{l}\text { Revisión Sistemática de estudios de } \\
\text { cohortes. }\end{array}$ \\
\hline & $2 \mathrm{~b}$ & $\begin{array}{l}\text { Estudios de cohortes individuales. } \\
\text { Ensayo Controlado de baja calidad. }\end{array}$ \\
\hline & $3 a$ & $\begin{array}{l}\text { Revisión Sistemática con } \\
\text { homogeneidad de estudios de caso y } \\
\text { controles. }\end{array}$ \\
\hline & $3 b$ & $\begin{array}{l}\text { Estudio de casos y controles } \\
\text { individuales. }\end{array}$ \\
\hline C & 4 & $\begin{array}{l}\text { Series de casos. Estudios de cohortes y } \\
\text { de casos y controles de mala calidad. }\end{array}$ \\
\hline $\mathrm{D}$ & 5 & $\begin{array}{l}\text { Opinión de expertos sin evaluación } \\
\text { crítica explícita, o basada en fisiología, } \\
\text { o en investigación teórica. }\end{array}$ \\
\hline
\end{tabular}

Como parte de las disciplinas insertas en el Sistema de Salud, la terapia ocupacional podría ser pertinente en el abordaje garantizado de los problemas de salud incorporados en el régimen GES. Actualmente, no se dispone de información que sistematice cómo la terapia ocupacional se incorpora en el plan GES, ni la evidencia disponible que sustente dicha incorporación a partir de los grados de recomendación existentes. Este análisis es relevante, debido a que los terapeutas ocupacionales perciben limitaciones en su capacidad de vincular la investigación científica con su práctica profesional (Graham, Robertson, y Anderson, 2013; Upton, Stephens, Williams y Scurlock-Evans, 2014), lo que de manera global se refleja en la limitada investigación científica que documente las decisiones clínicas y otorgue sustento a su efectividad, por lo que su escalamiento en los sistemas institucionales de protección a la salud se ve usualmente mermado (Aravena, 2015; Burke y Gitlin, 2012; Holm, 2000).

Por lo anterior, esta investigación tuvo por objetivo general caracterizar las prestaciones de terapia ocupacional incluidas en el régimen de Garantías Explícitas en Salud al año 2017, con los siguientes objetivos específicos: 1) Identificar las prestaciones de terapia ocupacional existentes en los 80 problemas de salud que incorpora el régimen de Garantías Explícitas en Salud, 2) Identificar los problemas de salud que incorporan atención de terapia ocupacional, y 3 ) Identificar los grados de recomendación referidos para las acciones de terapia ocupacional en los problemas de salud GES. Esta investigación busca aportar en la necesidad de evaluar el diálogo que la terapia ocupacional establece actualmente con directrices institucionales como ésta.

\section{MÉTOdo}

Para lograr el objetivo de este trabajo, se realizó una revisión documental, la que puede definirse como un proceso dinámico que consiste en la recogida, clasificación, recuperación y distribución de la información, cuyo propósito es brindar una descripción sobre la situación actual de algún problema o área (Rodríguez y Valldeoriola, 2009). La FIGURA 2 describe las etapas de la revisión documental, siguiendo las recomendaciones de Amador (1998), que considera que el proceso de revisión documental comprende tres etapas esenciales: consulta documental, contraste de la información y análisis histórico del problema. Fueron incluidas las siguientes fuentes de documentación primarias:

a. Guías Clínicas para problemas de salud GES, con un total de 8o. En estos documentos se realizó una búsqueda dirigida de las palabras integradas "terapeuta ocupacional" y "terapia ocupacional", y se registró el grado de recomendación y las acciones de terapia ocupacional descritas. Se consideró el documento disponible con la fecha más reciente de actualización.

b. Listado de Prestaciones Específicas. Corresponde a un documento que reúne las prestaciones codificadas, según catálogo de Fonasa, y que constituyen una garantía por ley. Se indagó manualmente y a través de búsqueda digital en prestaciones propias de terapia ocupacional con las palabras integradas "terapeuta ocupacional" $y$ "terapia ocupacional". 
Las fuentes de información fueron recopiladas entre los meses de marzo y julio 2017, desde el sitio web de la Superintendencia de Salud, Ministerio de Salud de Chiles.

FIGURA 2 PROCESO DE REVISIÓN DOCUMENTAL

Elaboración de dos matrices para recopilación de información de acuerdo con objetivos específicos. Matriz 1 para información de Guías Clínicas y Matriz 2 para información de Listado de Prestaciones Específicas.

Las dimensiones de información recopiladas incluyeron, para cada patología y fuente de información: Menciona la palabra "terapia ocupacional y/o terapeuta ocupacional (Sí/No), Descripción de la prestación (texto), grado de recomendación referido (A, B, C, D, Ninguno).

Recopilación de Guías Clínicas (80) Recopilación de Listados de Prestaciones Específicas (1)

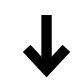

$1^{\text {era }}$ Consulta documental

Búsqueda de palabra clave "terapia ocupacional", y "terapeuta ocupacional". Asignación de 20 Guías Clínicas a cada investigador.

Además, cada investigador revisó de forma individual el $\mathrm{LP}_{3}$.

Vaciamiento de información en Matriz 1 y 2.

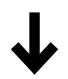

$2^{\text {a }}$ Consulta documental y contraste de información

Un segundo investigador revisó las fuentes de información de un grupo de 20 patologías diferentes a la 1a revisión para contrastar los hallazgos iniciales y completar la matriz definitiva.

\section{Resultados}

Los resultados de esta revisión documental se organizan a continuación según un objetivo específico.

\section{IDENTIFICACIÓN DE PRESTACIONES DE TERAPIA OCUPACIONAL INCORPORADAS EN EL RÉGIMEN GES}

La revisión documental del Listado de Prestaciones Específicas mostró la existencia de una prestación, denominada "Atención Integral por Terapeuta Ocupacional", con el código 102007.

Esta prestación se describe de la siguiente forma: "Prestación de salud que incluye por sesión, la evaluación de las actividades básicas de la vida diaria y los procedimientos terapéuticos en el ámbito de la rehabilitación funcional física y mental que realiza el terapeuta ocupacional a un paciente con prescripción médica para este tipo de tratamiento" (Resolución $\mathrm{N}^{\circ}$ 60, 2007).

\section{PROBLEMAS DE SALUD QUE INCORPORAN TERAPIA OCUPACIONAL}

La prestación "Atención integral por Terapeuta Ocupacional" es referida en 12 problemas de salud según el Listado de Prestaciones Específicas. Por su parte, en las Guías Clínicas se incorpora la terapia ocupacional en 10 problemas de salud, indicados en la TABLA 1, según su fuente de documentación.

5 http://www.supersalud.gob.cl 
TABLA 1 PROBLEMAS DE SALUD GES E INCORPORACIÓN DEL PROFESIONAL TERAPEUTA OCUPACIONAL, SEGÚN FUENTE DE DOCUMENTACIÓN.

\section{PROBLema DE SALUD}

1. Enfermedad renal crónica etapa 4 y 5

2. Cardiopatías congénitas operables en menores de 15 años

3. Cáncer Cérvico-uterino

4. Alivio del dolor y cuidador paliativos por cáncer avanzado

5. Infarto agudo del miocardio

6. Diabetes Mellitus tipo I

7. Diabetes Mellitus tipo II

8. Cáncer de mama en personas de 15 años o más

9. Disrafias espinales

10. Tratamiento quirúrgico de escoliosis en personas menores de 25 años

11. Tratamiento quirúrgico de cataratas

12. Endoprótesis total de cadera en personas de 65 años y más con artrosis de cadera con limitación funcional severa

13. Fisura Labiopalatina

14. Cáncer en personas menores de 15 años

15. Esquizofrenia

16. Cáncer de testículo en personas de 15 años y más

17. Linfomas en personas de 15 años y más

18. Síndrome de inmunodeficiencia adquirida VIH/SIDA

19. Infección respiratoria aguda (IRA) de manejo ambulatorio en personas menores de 5 años

20. Neumonía adquirida en la comunidad de manejo ambulatorio en personas de 65 años y más

21. Hipertensión arterial primaria o esencial en personas de 15 años y más

22. Epilepsia no refractaria en personas desde 1 año y menores de 15 años

23. Salud oral integral para niños y niñas de 6 años

24. Prevención de parto prematuro

25. Trastornos de generación del impulso y conducción en personas de 15 años y más, que requieren marcapaso

26. Colecistectomía preventiva del cáncer de vesícula en personas de 35 a 49 años

27. Cáncer gástrico

28. Cáncer de próstata en personas de 15 años y más

29. Vicios de refracción en personas de 65 años y más

30. Estrabismo en personas menores de 9 años

31. Retinopatía diabética

32. Desprendimiento de retina regmatógeno no traumático

33. Hemofilia 
PROBLEMA DE SALUD

34. Depresión en personas de 15 años y más

35. Tratamiento de la hiperplasia benigna de la próstata en personas sintomáticas

36. Órtesis (o ayudas técnicas) para personas de 65 años y más

37. Accidente cerebrovascular isquémico en personas de 15 años y más

38. Enfermedad pulmonar obstructiva crónica de tratamiento ambulatorio

39. Asma bronquial moderada y grave en menores de 15 años

40. Síndrome de dificultad respiratoria en el recién nacido

41. Tratamiento médico en personas de 55 años y más con artrosis de cadera y/o rodilla, leve o moderada

42. Hemorragia subaracnoidea secundaria a ruptura de aneurismas cerebrales

43. Tumores primarios del sistema nervioso central en personas de 15 años y más

44. Tratamiento quirúrgico de hernia del núcleo pulposo lumbar

45. Leucemia en personas de 15 años y más

46. Urgencia odontológica ambulatoria

47. Salud oral integral del adulto de 60 años

48. Politraumatizado grave

49. Traumatismo cráneo encefálico moderado o grave

50. Trauma ocular grave

51. Fibrosis quística

52. Artritis reumatoidea

53. Consumo perjudicial o dependencia de riesgo bajo a moderado de alcohol y drogas en personas menores de 20 años

54. Analgesia del parto

55. Gran quemado

56. Hipoacusia bilateral en personas de 65 años y más que requieren uso de audífono

57. Retinopatía del prematuro

58. Displasia broncopulmonar del prematuro

59. Hipoacusia neurosensorial bilateral del prematuro

60. Epilepsia no refractaria en personas de 15 años y más

61. Asma bronquial en personas de 15 años y más

62. Enfermedad de Parkinson

63. Artritis idiopática juvenil

64. Prevención secundaria enfermedad renal crónica terminal

65. Displasia luxante de caderas

66. Salud oral integral de la embarazada

67. Esclerosis múltiple remitente recurrente

68. Hepatitis crónica por virus hepatitis B

69. Hepatitis C 


\begin{tabular}{|c|c|c|}
\hline \multirow{2}{*}{ PROBLEMA DE SALUD } & \multicolumn{2}{|c|}{ FUENTE } \\
\hline & LPE & GC \\
\hline \multicolumn{3}{|l|}{ 70. Cáncer colorrectal en personas de 15 años y más } \\
\hline \multicolumn{3}{|l|}{ 71. Cáncer de ovario epitelial } \\
\hline \multicolumn{3}{|l|}{ 72. Cáncer vesicular en personas de 15 años y más } \\
\hline 73. Osteosarcoma en personas de 15 años y más & $\mathrm{X}$ & $X$ \\
\hline \multicolumn{3}{|l|}{$\begin{array}{l}\text { 74. Tratamiento quirúrgico de lesiones crónicas de la válvula aórtica en personas de } 15 \text { años y } \\
\text { más }\end{array}$} \\
\hline \multicolumn{3}{|l|}{ 75. Trastorno bipolar en personas de 15 años y más } \\
\hline \multicolumn{3}{|l|}{ 76. Hipotiroidismo en personas de 15 años y más } \\
\hline 78. Tratamiento de hipoacusia moderada en menores de 2 años & $\mathrm{X}$ & \\
\hline \multicolumn{3}{|l|}{ Lupus eritematoso sistémico } \\
\hline \multicolumn{3}{|l|}{$\begin{array}{l}\text { 79. Tratamiento quirúrgico de lesiones crónicas de las válvulas mitral y tricúspide en personas } \\
\text { de } 15 \text { años y más }\end{array}$} \\
\hline \multicolumn{3}{|l|}{ 80. Tratamiento de erradicación de helicobacter pylori } \\
\hline TOTAL & 12 & 10 \\
\hline
\end{tabular}

Puede observarse discrepancia entre la incorporación de terapia ocupacional según fuente de documentación. A continuación, la TABLA 2 describe los problemas de salud en los que no hay coincidencia entre las dos fuentes.
Según se observa en la tabla 2, existe una discrepancia entre la incorporación de terapia ocupacional según la fuente de documentación. Dicha situación podría explicarse por variados motivos. Por ejemplo, si se incluyó terapia ocupacional en un problema de salud declarado según LPE, pero no ha sido detallado

TABLA 2 PROBLEMAS DE SALUD GES CON DISCREPANCIA EN LA INCORPORACIÓN DE TERAPIA OCUPACIONAL SEGÚN FUENTE DE DOCUMENTACIÓN.

\begin{tabular}{|c|c|}
\hline Fuente de documentación & Problema de salud \\
\hline $\begin{array}{l}\text { Sólo en Listado de Prestaciones } \\
\text { Específicas }\end{array}$ & $\begin{array}{l}\text { 8. Cáncer de mama en personas de } 15 \text { años o más } \\
\text { 14. Cáncer en personas menores de } 15 \text { años } \\
\text { 15. Esquizofrenia } \\
\text { 59. Hipoacusia neurosensorial bilateral del prematuro } \\
\text { 78. Tratamiento de hipoacusia moderada en menores de } 2 \text { años }\end{array}$ \\
\hline Sólo en Guía Clínica & $\begin{array}{l}\text { 42. Hemorragia subaracnoidea secundaria a ruptura de aneurismas } \\
\text { cerebrales } \\
\text { 43. Tumores primarios del sistema nervioso central en personas de } 15 \\
\text { años y más } \\
\text { 62. Enfermedad de Parkinson }\end{array}$ \\
\hline
\end{tabular}


aún en su Guía Clínica, podría atribuirse a un desfase temporal entre la documentación administrativa y la documentación científica (que implica otros procesos de producción de información). Dicha situación podría también explicarse a partir de la ausencia de representación gremial en los procesos de elaboración de la Guía Clínica, considerando que las Guías de Práctica Clínica constituyen metodologías participativas de producción de información, que requieren el involucramiento de los diferentes actores que sean relevantes en el problema de salud que intenta abordarse.

El caso inverso (referencia de terapia ocupacional en Guía Clínica, pero no en LPE), debiese considerarse de mayor urgencia dado que se refiere la pertinencia técnica y científica de la profesión en un problema de salud determinado, pero no se declara la garantía de su cobertura administrativa y legal.

\section{GRADOS DE RECOMENDACIÓN SEGÚN PROBLEMA DE SALUD}

En el total de 10 problemas de salud cuyas Guías Clínicas incluyen la acción de terapia ocupacional, se indagó sobre el grado de recomendación referido y la descripción de las intervenciones de terapia ocupacional según evidencia (TABLA3). La información permite constatar que solamente 3 problemas de salud refieren Grado de Recomendación A (Accidente Cerebrovascular, Artritis Reumatoide, Gran Quemado) mientras las demás son informadas con Grados de Recomendación C y D, que corresponden a series de casos de baja calidad, investigación teórica y recomendación de experto. 
TABLA 3 GRADOS DE RECOMENDACIÓN Y DESCRIPCIÓN DE INTERVENCIONES DE TERAPIA OCUPACIONAL EN DIEZ PROBLEMAS DE SALUD GES

\begin{tabular}{|c|c|c|}
\hline PROBLEMA DE SALUD & GR & DESCRIPCIÓN \\
\hline Disrafias espinales & $\begin{array}{l}\text { No } \\
\text { referido }\end{array}$ & No disponible \\
\hline \multirow{2}{*}{$\begin{array}{l}\text { Accidente cerebrovascular } \\
\text { isquémico en personas de } 15 \\
\text { años y más }\end{array}$} & A & $\begin{array}{l}\text { Entrenamiento en AVD. } \\
\text { Incremento de participación en programas de tiempo libre. }\end{array}$ \\
\hline & $\mathrm{C}$ & Evaluación de conducción de automóvil \\
\hline $\begin{array}{l}\text { Hemorragia subaracnoidea } \\
\text { secundaria a ruptura de } \\
\text { aneurismas cerebrales }\end{array}$ & $\mathrm{C}$ & $\begin{array}{l}\text { Evaluación y apoyo a las personas con ACv que previamente } \\
\text { ejercían una labor. }\end{array}$ \\
\hline $\begin{array}{l}\text { Tumores primarios del } \\
\text { sistema nervioso central en } \\
\text { personas de } 15 \text { años y más }\end{array}$ & $\begin{array}{l}\text { No } \\
\text { referido }\end{array}$ & $\begin{array}{l}\text { La rehabilitación debe ser entregada por un equipo de tipo } \\
\text { interdisciplinario que puede contar con: médico fisiatra, médico } \\
\text { neurólogo, kinesiólogo, fonoaudiólogo, terapeuta ocupacional y } \\
\text { nutricionista. }\end{array}$ \\
\hline \multirow[t]{4}{*}{ Artritis reumatoidea } & A & $\begin{array}{l}\text { Se recomienda que todo paciente con AR tenga acceso a } \\
\text { sesiones anuales de tratamiento con terapeuta ocupacional. En } \\
\text { ellas se debiesen reforzar aspectos educativos de protección } \\
\text { articular aplicada a las rutinas y actividades cotidianas, } \\
\text { adecuaciones ergonómicas y del puesto de trabajo. }\end{array}$ \\
\hline & $A$ & $\begin{array}{l}\text { Se recomienda que todo paciente con AR sea derivado a } \\
\text { rehabilitación integral con fisiatra, kinesiólogo y terapeuta } \\
\text { ocupacional. }\end{array}$ \\
\hline & $\mathrm{C}$ & $\begin{array}{l}\text { Se recomienda la prescripción y entrenamiento en uso de órtesis } \\
\text { o férulas que puedan ser necesarias para el mejoramiento de la } \\
\text { calidad de vida de estos pacientes. }\end{array}$ \\
\hline & $\mathrm{C}$ & $\begin{array}{l}\text { De tener la posibilidad, se recomienda que los pacientes con AR } \\
\text { sean derivados a un Centro de Rehabilitación con kinesiólogo y } \\
\text { terapeuta ocupacional. }\end{array}$ \\
\hline Gran quemado & A & $\begin{array}{l}\text { Las prendas elásticas compresivas deben ser confeccionadas a } \\
\text { medida por kinesiólogos o terapeutas ocupacionales y deben ser } \\
\text { controlados en forma periódica durante el tiempo que dure el } \\
\text { tratamiento. Puede además ser necesario adicionar insertos para } \\
\text { optimizar la presión en áreas específicas. }\end{array}$ \\
\hline Enfermedad de Parkinson & $\begin{array}{l}\text { No } \\
\text { referido }\end{array}$ & $\begin{array}{l}\text { El principal objetivo de la terapia ocupacional es capacitar a } \\
\text { las personas para participar en las actividades de la vida diaria. } \\
\text { La terapia ocupacional es un proceso complejo que implica la } \\
\text { interacción cooperativa entre el profesional y la persona, inmersa } \\
\text { en el contexto de intervención. Se plantean } 3 \text { principios que } \\
\text { guían la terapia ocupacional contemporánea: la práctica centrada } \\
\text { en la persona, en la ocupación y en la evidencia. La intervención } \\
\text { de terapia ocupacional se organizará en tres momentos, según } \\
\text { la progresión de la enfermedad, determinado por la escala de } \\
\text { Hoehn y Yahr. }\end{array}$ \\
\hline
\end{tabular}




\begin{tabular}{|c|c|c|}
\hline PROBLEMA DE SALUd & GR & DESCRIPCIÓN \\
\hline & & 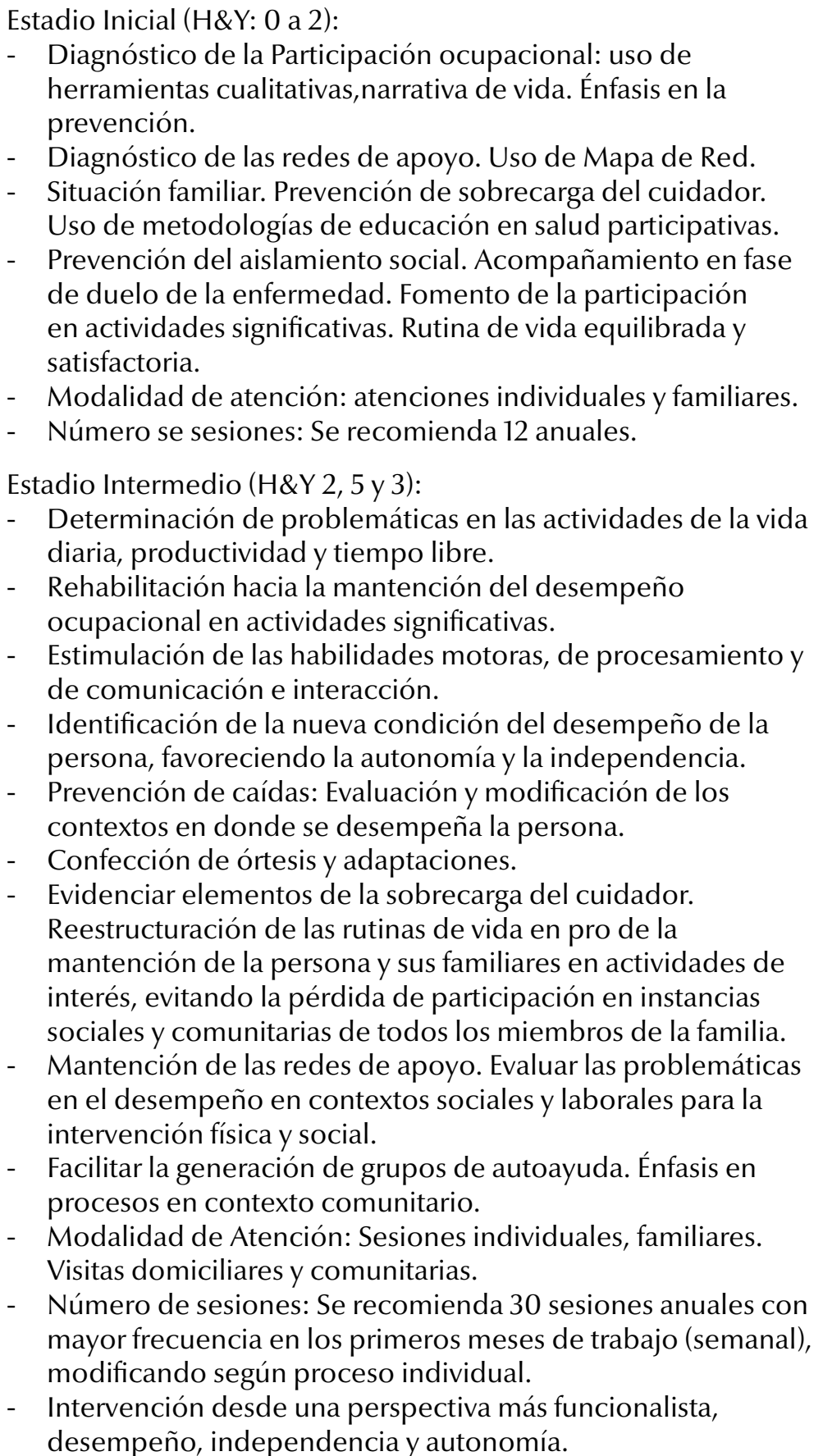 \\
\hline
\end{tabular}




\begin{tabular}{|c|c|c|}
\hline PROBLEMA DE SALUD & GR & DESCRIPCIÓN \\
\hline & & $\begin{array}{l}\text { Estadio Avanzado (H\&Y: } 4 \text { a 5): } \\
\text { - Énfasis de la intervención en el cuidador(a), para el manejo } \\
\text { de la persona afectada en pro de la calidad de vida del grupo } \\
\text { familiar. } \\
\text { - } \quad \text { Evaluación del desempeño ocupacional de la persona (uso } \\
\text { pauta sugerida que puede ser respondida por el cuidador(a)). } \\
\text { - } \quad \text { Determinación de áreas críticas. } \\
\text { - } \quad \text { Evaluación de sobrecarga del cuidador (Zarit). } \\
\text { - Entrenamiento de la asistencia en las actividades de la vida } \\
\text { diaria, favoreciendo el máximo de independencia posible } \\
\text { (colaboración, elección en ciertos pasos de la tarea). } \\
\text { - Prevención de lesiones por sobrecarga del cuidador (a), } \\
\text { educación postural, manejo ambiental, uso de barras, } \\
\text { elementos de transferencia, adaptaciones en baño, } \\
\text { dormitorios, etc. } \\
\text { - Estructuración de rutina de vida, procurando espacios de ocio } \\
\text { y tiempo libre para el cuidador (a). Uso de redes de apoyo } \\
\text { familiares y comunitarias. } \\
\text { - Modalidad de atención: Sesiones individuales, familiares y } \\
\text { visitas domiciliarias. } \\
\text { - Número de Sesiones: Se recomienda } 30 \text { sesiones anuales } \\
\text { dependiendo del nivel de sobrecarga. }\end{array}$ \\
\hline \multirow[t]{2}{*}{ Artritis idiopática juvenil } & $\mathrm{C}$ & $\begin{array}{l}\text { Todo paciente con AlJ/ARJ debe recibir tratamiento con terapeuta } \\
\text { ocupacional, haciendo hincapié en protección articular, así } \\
\text { como elaboración de órtesis y ayudas técnicas que puedan ser } \\
\text { necesarias. }\end{array}$ \\
\hline & $\mathrm{C}$ & $\begin{array}{l}\text { Rehabilitación Integral. Todo paciente con AIJ/ARJ deberá } \\
\text { recibir indicaciones de rehabilitación integral, realizado por } \\
\text { equipo especializado (kinesioterapia, fisioterapia, ejercicios, } \\
\text { terapia ocupacional), apoyado eventualmente por profesor } \\
\text { de educación física, nutricionista y psicólogo, lo cual debe ser } \\
\text { personalizado de acuerdo a las características particulares de su } \\
\text { enfermedad. }\end{array}$ \\
\hline $\begin{array}{l}\text { Esclerosis múltiple remitente } \\
\text { recurrente }\end{array}$ & $\mathrm{C}$ & $\begin{array}{l}\text { El equipo tratante debe, en lo posible, estar integrado por } \\
\text { neurólogo, fisiatra, psicólogo, enfermera, kinesiólogo, terapeuta } \\
\text { ocupacional, asistente social, fonoaudiólogo y nutricionista con } \\
\text { un enfoque multidisciplinario. }\end{array}$ \\
\hline $\begin{array}{l}\text { Osteosarcoma en personas } \\
\text { de } 15 \text { años y más }\end{array}$ & $\mathrm{D}$ & $\begin{array}{l}\text { La mayoría de los autores sugiere que la rehabilitación que } \\
\text { incluye kinesiterapia, terapia ocupacional y adaptación } \\
\text { psicológica está indicada especialmente para el entrenamiento } \\
\text { de la colocación de prótesis y entrenamiento de su uso cuando } \\
\text { se trata de cirugía radicular o la aplicación de protocolos } \\
\text { kinésicos específicos cuando se trata de cirugía conservadora, lo } \\
\text { que mejora la calidad de vida y funcionalidad de los pacientes. }\end{array}$ \\
\hline
\end{tabular}




\section{Discusión}

Esta investigación tuvo por propósito caracterizar la incorporación de terapia ocupacional en el actual régimen de Garantías Explícitas en Salud (GES). Esta modalidad de atención institucional representa una las reformas más relevantes en salud que por ley asegura estándares de calidad, acceso y protección económica para el tratamiento de 80 problemas de salud. En este contexto, resulta altamente necesario evaluar el diálogo que la terapia ocupacional establece actualmente con esta definición institucional.

Entre sus hallazgos, esta investigación documenta la existencia de una prestación de terapia ocupacional, denominada "Atención integral por terapeuta ocupacional", la cual cuenta con una descripción en la Resolución $N^{\circ} 6$ o que aprueba normas de carácter técnico médico y administrativo para el cumplimiento de las garantías explícitas en salud de la ley 19.966. Ante la actual iniciativa de incorporación de prestaciones de terapia ocupacional en la cartera del Fonasa, resulta pertinente resaltar la existencia de dicha prestación ya incorporada en este seguro de salud, lo que podría constituir una primera etapa para su transferencia a la Modalidad de Libre Elección.

En el total de 8o problemas de salud cubiertos por GES, existe una discrepancia entre las dos fuentes de documentación consultadas. El Listado de Prestaciones Específicas incluye la terapia ocupacional en 12 problemas de salud, mientras que las Guías Clínicas la incorporan en 10. Dicha discrepancia puede deberse a que la temporalidad del Listado de Prestaciones Específicas puede no coincidir con el de las Guías Clínicas, dado que varias de éstas no han sido actualizadas.

Los Grados de Recomendación de las intervenciones de terapia ocupacional son en su mayoría C y D, representando grados iniciales de recomendación y evidencia. Actualmente el estado del arte sobre la efectividad de la terapia ocupacional corresponde a niveles incipientes de desarrollo de evidencia. De lo contrario, asumiendo que existiese mejor evidencia disponible, los bajos grados de recomendación podrían explicarse por una menor rigurosidad en la revisión de evidencia para el desarrollo de las guías clínicas respectivas. Cualquiera sea el escenario, se identifica una oportunidad, en un escenario global en el que la práctica guiada por evidencia toma progresivamente mayor relevancia (Ottenbacher, Tickle-Degnen \& Hasselkus, 2002).

A partir de la revisión, pudo constatarse que la terapia ocupacional se encuentra garantizada en 12 de los 80 problemas de salud de GES. Podría pensarse que este número es bajo en proporción, no obstante, dicha valoración debe hacerse con responsabilidad a la luz de la evidencia disponible. Como ejemplo, esta revisión constató que el problema de salud $\mathrm{N}^{\circ} 36$ "Órtesis (o ayudas técnicas) para personas de 65 años y más" no incorpora la acción del terapeuta ocupacional, siendo ésta un componente de intervención frecuente en la acción y formación profesional, y en la que existe evidencia creciente de su efectividad a partir de ensayos controlados aleatorizados, revisiones sistemáticas y estudios de costo efectividad (Clark et al., 2012; De Coninck et al., 2017; Zingmark, Nilsson, Norström, Sahlén \& Lindholm, 2017).

Lo anterior es pertinente a otros problemas de salud, permitiendo entonces plantear interrogantes sobre los focos de investigación que se desarrollan actualmente en la disciplina. ¿Estamos priorizando investigación útil a la toma de decisiones y a los problemas de salud de mayor impacto en nuestro país? Dada la relevancia que tiene el sistema de GES, esta modalidad institucional debiese constituir un área de interés prioritaria para la investigación en terapia ocupacional.

Esta investigación permitió constatar que algunas Guías Clínicas incorporan de manera genérica la acción de terapeuta ocupacional, refiriendo altos grados de recomendación para el trabajo multidisciplinar que incorpore la profesión, sin detallar con precisión cuáles son las intervenciones recomendadas. Lo anterior puede observarse, por ejemplo, en la Guía Clínica de Disrafia Espinal. Asimismo, es posible observar otras guías que sí describen de manera detallada ciertas intervenciones sugeridas; no obstante, no explicitan los niveles de evidencia ni los grados de recomendación consiguientes que sustenten dichos planteamientos técnicos. Un ejemplo de lo anterior es lo reportado en la Guía Clínica de Parkinson, lo cual es contrastante con la evidencia disponible que señala que no existe evidencia clara que permita recomendar o no recomendar la intervención de terapia ocupacional en dicho problema de salud (Rao, 2010).

A partir de lo anterior, cabe preguntarse, ¿cuáles son los problemas de salud GES en los que la terapia ocupa- 
cional tendría pertinencia técnica y científica y debiese ser garantizada por ley? Esta pregunta permite problematizar cómo se incorpora actualmente la evidencia en la formación de terapeutas ocupacionales en Chile, y al mismo tiempo, cómo se asume de manera gremial la necesidad de promover prácticas guiadas por evidencia, traduciendo el conocimiento a las prácticas en contexto, y abordando la gestión estratégica del diálogo con las instituciones del aparato estatal.

La presente investigación abre la posibilidad de nuevos estudios que profundicen en el estado de la evidencia actual para cada uno de los 80 problemas de salud GES, y también de otros. Por ejemplo, existen revisiones sistemáticas que informan de la efectividad de terapia ocupacional para personas con demencias y sus entornos de apoyo (Laver et al., 2017; Livingston et al., 2017), enfermedades musculoesqueléticas (Marik \& Roll, 2017), cáncer (Hunter, Gibson, Arbesman \& D'Amico, 2017), Artritis Reumatoide (Siegel, Tencza, Apodaca \& Poole, 2017), y delirium (Álvarez et al., 2017). Si se comprende adecuadamente el modelo de jerarquía de evidencia, el análisis de evidencia disponible no debiese considerarse un método taxativo de definición de las prácticas de terapia ocupacional en salud, sino como un estudio sistemático que permite informar la toma de decisiones y levantar otras áreas de problematización. Por ejemplo, revisiones sistemáticas que han evaluado la efectividad de intervenciones han incluido en sus hallazgos que muchas de las acciones de terapia ocupacional no corresponden a estrategias con base en la ocupación (Kadar, Mcdonald \& Lentin, 2012), y otras han posibilitado problematizar la elección de resultados que puedan informar de los beneficios de la terapia ocupacional, focalizando entonces en la metodología de las sistematización de práctica (Steultjens et al., 2003). Esta información es fundamental si se busca delimitar los campos de acción, los riesgos y los beneficios esperados de la profesión, lo que constituye, por sobre todo, una necesidad ética.

Puede mencionarse que el Listado de Prestaciones Específicas incluye prestaciones genéricas, tales como "Consulta de salud mental por otros profesionales (ogozo03)", e "Intervención psicosocial grupal (4 a 8 pacientes, familiares o cuidadores; 0903004)". Es posible que en la actualidad dichas prestaciones incluyan al profesional de terapia ocupacional, lo cual no fue incorporado en esta revisión, y podría abordarse a través de la revisión de registros estadísticos y así ampliar el análisis propuesto en esta investigación.
El sistema de Garantías Explícitas en Salud constituye una de las iniciativas más relevantes de reforma del Sistema de Salud Chileno, según su alcance poblacional y sus premisas de equiparación de brechas de acceso y desigualdad en resultados en salud. Por lo anterior, la terapia ocupacional debe potenciar su diálogo con esta modalidad de Salud Pública, reforzando así la urgente necesidad de continuar construyendo la evidencia científica que enmarque su acción y establezca una pertinente mayor cobertura y acceso a las personas.

\section{ReferenCiAs BibliográfiCAS}

Álvarez, E. A., Garrido, M. A., Tobar, E. A., Prieto, S. A., Vergara, S. O., Briceño, C. D., \& González, F. J. (2017). Occupational therapy for delirium management in elderly patients without mechanical ventilation in an intensive care unit: A pilot randomized clinical trial. Journal of Critical Care, 37, 85-90. http://doi.org/10.1016/j. jcrc.2016.09.002

Aravena, J. M. (2015). ¿Qué tanto entendemos el concepto de práctica basada en la evidencia en terapia ocupacional? Revista Chilena de Terapia Ocupacional, 15(1), 189-192.

Bastías, G., Pantoja, T., Leisewitz, T. \& Zárate, V. (2008). Health care reform in Chile. Canadian Medical Association Journal, 179(12), 1289-1292. http://doi.org/10.1503/cmaj.071843

Burke, J. P. \& Gitlin, L. N. (2012). How Do We Change Practice When We Have the Evidence? American Journal of Occupational Therapy, 66, e85-e88. http://doi.org/10.5014/ajot.2012.004432

Clark, F., Jackson, J., Carlson, M., Chou, C., Cherry, B. J., Jordan-marsh, M., ... Azen, S. P. (2012). Effectiveness of a lifestyle intervention in promoting the well-being of independently living older people: results of the Well Elderly 2 Randomised Controlled Trial.Journal of Epidemiology and Community Health, 66, 182-790. http://doi. org/10.1136/jech.2009.099754

De Coninck, L., Bekkering, G. E., Bouckaert, L., Declercq, A., Graff, M. J. L. \& Aertgeerts, B. (2017). Home- and Community-Based Occupational Therapy Improves Functioning in Frail Older People: A Systematic Review. Journal of the American Geriatrics Society, 65(8), 1863-1869. http://doi.org/10.1111/jgs.14889

Graham, F., Robertson, L. \& Anderson, J. (2013). New Zealand occupational therapists' views on evidence-based practice: A replicated survey of attitudes, confidence and behaviours. Australian Occupational Therapy Journal, 60(2), 120-128. http:// doi.org/10.1111/1440-1630.12000

Henríquez, J. (2017). Limitaciones al acceso de las Garantías Explícitas en Salud (GES). Centro de Estudios Públicos. Puntos de Referencia, (458), 1-10.

Holm, M. B. (2000). Our Mandate for the New Millennium: Evidence-Based Practice, 2000 Eleonor Clarke Slagle lecture. 
American Journal of Occupational Therapy, 54(607), 575-585. http://doi.org/10.5014/ajot.54.6.575

Hunter, E. G., Gibson, R. W., Arbesman, M. \& D'Amico, M. (2017). Systematic review of occupational therapy and adult cancer rehabilitation: Part 1. Impact of physical activity and symptom management interventions. American Journal of Occupational Therapy, 71(2). http://doi.org/10.5014/ajot.2017.023564

Kadar, M., Mcdonald, R. \& Lentin, P. (2012). Evidence-based practice in occupational therapy services for children with autism spectrum disorders in Victoria, Australia. Australian Occupational Therapy Journal, 59(4), 284-293. http://doi.org/10.1111/j.14401630.2012.01015.x

Laver, K., Cumming, R., Dyer, S., Agar, M., Anstey, K. J., Beattie, E., ... Yates, M. (2017). Evidence-based occupational therapy for people with dementia and their families: What clinical practice guidelines tell us and implications for practice. Australian Occupational TherapyJournal, 64(1), 3-10. http://doi.org/10.1111/1440-1630.12309

Livingston, G., Sommerlad, A., Orgeta, V., Costafreda, S. G., Huntley, J., Ames, D., ... Mukadam, N. (2017). Dementia prevention, intervention, and care. The Lancet. http://doi.org/http://dx.doi.org/10.1016/ S0140-6736(17)31363-6 See

Manterola, C., Asenjo-Lobos, C. \& Otzen, T. (2014). Jerarquización de la evidencia. Niveles de evidencia y grados de recomendación de uso actual. Revista Chilena de Infectología, 37(6), 705-18. http:// doi.org/10.4067/S0716-10182014000600011

Marik, T. L. \& Roll, S. C. (2017). Effectiveness of occupational therapy interventions for musculoskeletal shoulder conditions: A systematic review. American Journal of Occupational Therapy, 71(1). http://doi.org/10.5014/ajot.2017.023127

Ministerio de Salud. Aprueba normas de carácter técnico, médico, y administrativo para el cumplimiento de las Garantías Explícitas en Salud de la Ley 19.966 (2013).

Ministerio de Salud. (2014). Manual metodológico Desarrollo de Guías de Práctica Clínica. Recuperado de http://www.bibliotecaminsal. $\mathrm{cl} / \mathrm{wp} / \mathrm{wp}$-content/uploads/2016/04/Manual-metodologico-GPC-151014.pdf

Ministerio de Salud. Listado de prestaciones específicas (2016). Anexo decreto Régimen de Garantías Explícitas en Salud. Recuperado de http://www.minsal.cl/portal/url/item/d692c627c658b9cae040010164016563.pdf

Ottenbacher, K. J., Tickle-Degnen, L. \& Hasselkus, B. R. (2002). Therapists awake! The challenge of evidence-based occupational therapy. The American Journal of Occupational Therapy, 56(3), 247-249. http://doi.org/10.5014/ajot.56.3.247

Pantoja, T. \& Soto, M. (2014). Guías de práctica clínica: una introducción a su elaboración e implementación. Revista Médica de Chile, 142, 98-104.

Pardo, C. \& Schott, W. (2014). Health insurance selection in Chile: a cross-sectional and panel analysis. Health Policy and Planning, 29(3), 302-312. http://doi.org/10.1093/heapol/czt017
Rao, A. K. (2010). Enabling functional independence in Parkinson's disease: Update on occupational therapy intervention. Movement Disorders, 25(SUPPL. 1), 146-151. http://doi.org/10.1002/mds.22784

Resolución Nº6 (2007). Aprueba normas de carácter técnico médico y administrativo para el cumplimiento de las garantías explícitas en salud de la Ley $N^{\circ}$ 19.966. Ministerio de Salud. Diario Oficial de la República de Chile.

Rodríguez, D. \& Valldeoriola, J. (2009). Metodología de la investigación. Metodología de la Investigación. Universidat Overta de Catalunya: Material docente de la UOC. Recuperado de http://www.casadellibro.com/libro-metodologia-de-la-investigacion-5-ed-incluye-cd-rom/9786071502919/1960006

Sackett, D. L. (1986). Rules of Evidence and Clinical Recommendations on the Use of Antithrombotic Agents. CHEST, 89(2), 2S-3S. http:// doi.org/10.1378/chest.89.2

Siegel, P., Tencza, M., Apodaca, B. \& Poole, J. L. (2017). Effectiveness of occupational therapy interventions for adults with rheumatoid arthritis: A systematic review. American Journal of Occupational Therapy, 71(1). http://doi.org/10.5014/ajot.2017.023176

Steultjens, E. M., Dekker, J., Bouter, L. M., Cardol, M., Van de Nes, J. C., Van den Ende, C. H., ... Van den Ende, C. H. M. (2003). Occupational therapy for multiple sclerosis. Cochrane Database of Systematic Reviews, (3), CD003608. http://doi.org/10.1002/14651858. CD003608

Upton, D., Stephens, D., Williams, B. \& Scurlock-Evans, L. (2014). Occupational therapists' attitudes, knowledge, and implementation of evidence-based practice: A systematic review of published research. British Journal of Occupational Therapy, 77(1), 24-38. http://doi.org/10.4276/030802214X13887685335544

Zingmark, M., Nilsson, I., Norström, F., Sahlén, K. G. \& Lindholm, L. (2017). Cost effectiveness of an intervention focused on reducing bathing disability. European Journal of Ageing, 14, 233-241. http:// doi.org/10.1007/s10433-016-0404-1 
